\section{Prescription of atropine eye drops among children diagnosed with myopia in Taiwan from 2000 to 2007: a nationwide study}

${ }^{1}$ Institute of Health and Welfare Policy, National Yang-Ming University, Taipei, Taiwan

${ }^{2}$ Universal Eye Center, Taoyuan Branch, Taoyuan, Taiwan

${ }^{3}$ Institute of Public Health and Community Medicine Research Center, National Yang-Ming University,

Taipei, Taiwan

${ }^{4}$ Institute of Hospital and Health Care Administration, School of Medicine, National Yang-Ming University, Taipei, Taiwan

${ }^{5}$ Universal Eye Center, Taipei Branch, Taipei, Taiwan

${ }^{6}$ Department of Ophthalmology and Optometry, Fujian Medical University, Fuzhou, China

${ }^{7}$ Department of Health Policy and Management, University of North Carolina at Chapel Hill, Chapel Hill, NC, USA

Correspondence:

C Pu, Institute of Hospital and Health Care

Administration, School of Medicine, National Yang-Ming University, No. 155, Li-Nong Street, Sec. 2, Peitou, Taipei 112, Taiwan. Tel: + 8862 28267942; Fax: + 8862 28261002; E-mail: cypu@ym.edu.tw

Received: 12 May 2011 Accepted in revised form: 23 November 2012 Published online: 4 January 2013

\begin{abstract}
Purpose This study was conducted to examine the atropine eye drop prescription trend for children diagnosed with myopia, and to determine the factors associated with the prescription of atropine eye drops. Design This was a population-based cross-sectional study.

Methods This study was conducted using a national representative sample from the National Health Insurance (NHI) claims data. All school children between 4 and 18 years of age who had visited an ophthalmologist and were diagnosed with myopia between 2000 and 2007 were included herein. The main outcome measure was the proportion of subjects who were prescribed atropine eye drops in each year. Logistic regression was used to identify the factors associated with atropine eye drops being prescribed.

Results The prescription of atropine eye drops for children diagnosed with myopia increased significantly from the school years $2000(36.9 \%)$ to $2007(49.5 \%)$. There was also a shift from prescribing high concentrations ( 0.5 and $1 \%)$ of atropine eye drops to lower concentration ones $(0.3,0.25$, and $0.1 \%)$ within this period. Atropine eye drops were more frequently prescribed to 9-12-year-old children $(O R=1.26-1.42$, compared with those 7-8 years old), and to children from families with a high socioeconomic status $(O R=1.19-1.25)$; however, they were less prescribed to those living in mid to low urbanized areas $(\mathrm{OR}=\mathbf{0 . 6 5 - 0 . 8 4 )}$. Conclusions This study revealed an increasing trend of atropine eye drop prescription for children with myopia in
\end{abstract}

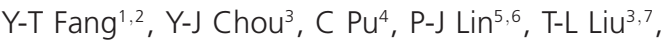
$\mathrm{N} \mathrm{Huang}{ }^{4}$ and $\mathrm{P} \mathrm{Chou}^{3}$

Taiwan. Our study provides eye-care professionals worldwide a reference for the potential integration of atropine eye drops into their clinical practice toward children with myopia.

Eye (2013) 27, 418-424; doi:10.1038/eye.2012.279; published online 4 January 2013

Keywords: atropine; myopia; prescription; National Health Insurance claim data

\section{Introduction}

Myopia is an important health concern in many East Asian ${ }^{1-3}$ and Western countries. ${ }^{4-6}$

In Taiwan, the prevalence of myopia among 12and 13-year-old children is among the highest in South-East Asia. ${ }^{1,3,7}$ A trend toward increasing myopia among 7-18-year-old Taiwanese children was observed between 1983 and 2000. ${ }^{8}$ A similar but less extreme trend was found for 12-17 year olds in the United States, depicting an increase from $25 \%$ in $1971-1972$ to $41.6 \%$ in 1999-2004..$^{5}$ Given the increasing prevalence and severity of myopia among children worldwide, a variety of interventions have been developed to retard the progression of myopia. ${ }^{9,10}$ Most of the proposed interventions lack conclusive evidences to support their effectiveness. ${ }^{10}$

Atropine is a nonselective muscarinic receptor antagonist, which is used to retard myopia progression among children in South Eastern Asia. Atropine has become part of routine eye care in Taiwan ever since the suggestion by the Taiwan Ophthalmology Association in the year 2000. Although evidences from many clinical trials on the use of 
atropine eye drops support a statistically significant reduction in myopia progression between experimental and control groups, ${ }^{9-16}$ the clinical significance of the results of these trials are controversial. The routine use of atropine among children with myopia is not recommended in other countries. ${ }^{10}$

Aside from the issue of effectiveness, some ophthalmologists are concerned about the possible longterm side effects from the use of atropine eye drops, including phototoxic effects on the retina and lens. ${ }^{10}$ Others are concerned about near vision blurring, photophobia, and allergic reaction, ${ }^{12}$ which could be lessened by atropine eye drops of lower concentrations. In treating myopia among children, $0.5 \%$ atropine is more effective than 0.25 and $0.1 \%$ atropine. ${ }^{12}$ One study found that the clinical effectiveness of higher concentrations of atropine eye drops is only slightly higher than lower concentration ones in slowing myopia progression among children, although the difference achieved statistical significance. ${ }^{17}$ However, treatments with higher concentrations of atropine eye drops are associated with a higher rate of dropout, higher level of photophobia due to larger pupil size, and lower amplitude of accommodation. ${ }^{12,17}$

The goal of our study is to investigate the prescription pattern of atropine eye drops among children with myopia in Taiwan from 2000 to 2007. Taiwanese ophthalmologists commonly use atropine as a treatment modality in their daily practice. As no other recommendations about myopia treatment have been proposed, it is important to determine the trends in prescribing atropine by ophthalmologists in Taiwan. This is because not only do they face one of the highest percentages of children with myopia, but they also serve a population with a high progression rate. ${ }^{18}$ This study does not intend to discuss whether atropine should be prescribed to retard the progression of myopia. As atropine is only one of the methods available for retarding the progression of myopia, other modalities should also be considered in clinical practice. Whether or not to adopt atropine depends not only on the effectiveness of the treatment and the side effects, but also on various other patient and environmental factors.

\section{Materials and methods}

\section{Data}

The National Health Insurance Research Database (NHIRD) consists of all administrative records of healthcare utilization under the National Health Insurance (NHI) program in Taiwan. Enrollment in the NHI is compulsory for all citizens, with a coverage rate of over
99\%. The NHI covers all outpatient and inpatient visits. The data used in this study consist of one million randomly selected samples from all citizens for the period from 1 January to 31 December 2005. All citizens had an equal chance of being selected into the study sample. This data set is linked to all medical utilization records of these samples, retrospectively from 2000 and prospectively to 2008 (which represents the school years 2000-2007). A detailed description of the sampling method can be found elsewhere. ${ }^{19,20}$ The National Health Research Institutes scrambles the individual IDs before providing the data to scientists in Taiwan for research purposes to ensure patient privacy. The data set is divided according to school year rather than calendar year because of the high correlation between visiting an ophthalmologist and the annual vision screening conducted in the schools. In this study, the school year runs from 2 September of one year to 1 September of the next year.

\section{Study population}

During each school year, we examined individuals aged between 4 and 18 years to check if they were diagnosed with myopia. To maintain homogeneity and ensure that our analysis included only those patients who were prescribed atropine eye drops for myopia treatment, individuals with an International Classification of Diseases, Ninth Clinical Modification diagnosis (ICD-9 CM) of anterior or pan-uveitis (ICD 360.0, 360.1, 091.5, 091.50, 364 (excluding 364.5, 364.6), 053.22, 054.44, 091.52, 098.41), accommodative esotropia (ICD 378.35), malignant glaucoma (ICD 365.83), and inflammatory glaucoma (ICD 365.62) were excluded (669 children, $0.03 \%$ ). Those being prescribed atropine ointment were excluded (1258 children, $0.06 \%)$. In addition, individuals with incomplete data (6253 children, $0.28 \%$ ) were also excluded.

Myopia is defined using ICD-9-CM as ICD 367. We used ICD 367 instead of the more specific diagnosis of 367.1 for myopia, as physicians normally categorize myopia diagnosis under category 367 , and thus limiting the diagnoses to 367.1 would exclude a number of children who were considered having myopia. In this study, only $62.9 \%$ of children who were prescribed atropine eye drops were diagnosed under category 367.1, and thus if we use specific diagnosis, approximately onethird of children being prescribed atropine would be excluded from this study. By using ICD 367 to define myopia, $89.5 \%$ of all children prescribed atropine eye drops would be included. Sensitivity analysis showed that the results are similar for these two definitions of myopia diagnosis (table not shown). 


\section{Study variables}

The school grade of the pupil was calculated from their birthday for each of the school years being analyzed. Grade 1 pupils in elementary school were assigned to be age 7 years, whereas those in grade 6 in elementary schools were 12 years old. Children were categorized into six age groups, corresponding to the ages in kindergarten (4-6 years old), elementary school $(7-8,9-10$, and 11-12 years old), junior high school (13-15 years old), and senior high school (16-18 years old). The level of urbanization was determined by the residential area code of the subjects. There are 361 different administrative areas within Taiwan. They are grouped into three strata according to their level of urbanization, based on their administrative classification. Level 1 includes metropolitan cities such as Taipei City, Taichung City, and Kaohsiung City. Level 2 includes other cities and counties. Level 3 consists of townships and represents any rural areas.

Socioeconomic status (SES) was classified according to the monthly wage of the child's parent or guardian and was divided into three categories, namely NTD $\geq 40000$, NTD 20 000-39999 NTD, and NTD <20000 (1 USD = 30 NTD in May 2012). Those without a well-defined monthly wage were categorized into two groups: first farmers and fishermen and second low-income individuals enrolled in the NHI through local government offices. The latter consists of mostly unemployed individuals.

Five different concentrations of atropine eye drops are currently reimbursed under the NHI program. The 0.5 and $1 \%$ atropine formulations have been available for reimbursement since 1995, the $0.3 \%$ formulation since 2001, and the $0.1 \%$ formulation since 2004 .

\section{Statistical analysis}

The unit of observation was the individual child during each school year. We first analyzed the trend of ophthalmology visits among 4-18 year olds during the study period (Figure 1). From the children who visited ophthalmologists and had a diagnosis of ICD-9 CM 367, we analyzed the proportion who were being prescribed atropine eye drops (Figure 2). The trend of the various concentrations being prescribed is shown in Figure 3. The proportion of atropine prescribed was calculated by dividing 'the number of subjects with a myopia diagnosis who had received at least one prescription for atropine eye drops in each analytic stratum during a specific school year' by 'the total number of subjects with a myopia diagnosis in each analytic stratum during a specific school year'. We applied multiple logistic regression analysis to determine the factors associated

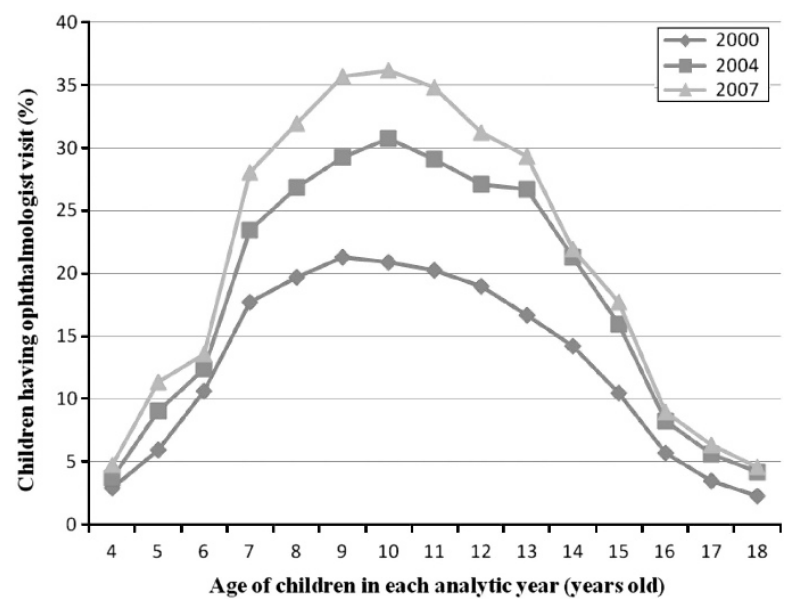

Figure 1 Children who visited ophthalmologist (\%) during the school years 2000, 2004, and 2007. For all 4- to 18-year-old children, the percentage of children who visited ophthalmologist had significantly increased from $12.7 \%$ in 2000 to $18.5 \%$ in 2004 and to $21.4 \%$ in 2007. For 10-year-old children, the percentage of children who visited ophthalmologist had significantly increased from $20.9 \%$ in 2000 to $30.7 \%$ in 2004 and to $36.1 \%$ in 2007.

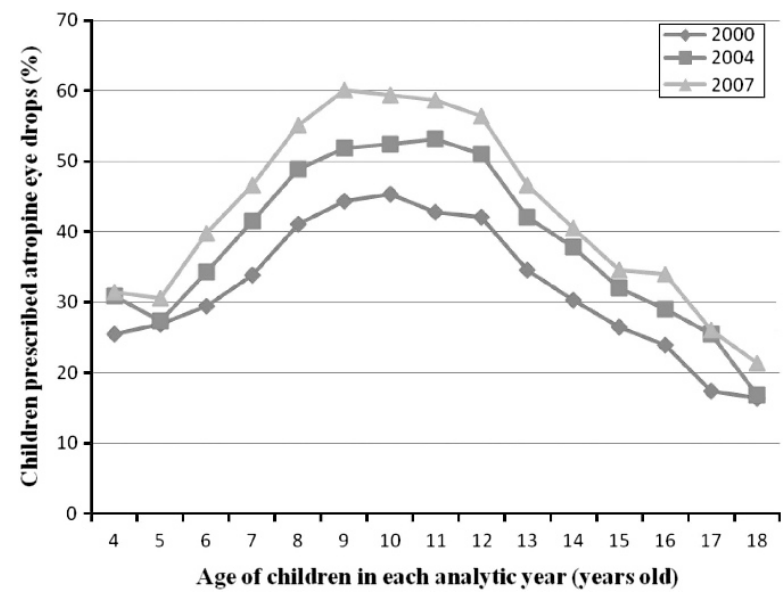

Figure 2 Atropine eye drop prescription (\%) among children diagnosed with myopia.

with the prescription of atropine eye drops. A subject was coded 1 if $s /$ he had ever been prescribed atropine during the study period, and 0 otherwise.

\section{Results}

There was an overall $68.3 \%$ increase in ophthalmologist visits for 4-18 year olds from 2000 to 2007 (see Figure 1) Among them, 85\% (85.1, 86.2, and 87.8\% in 2000, 2004, and 2007, respectively) were diagnosed as 'Disorders of refraction and accommodation' (ICD-9 CM 367). There was no difference among the different age groups. Children who were diagnosed with ICD-9 367 showed an 


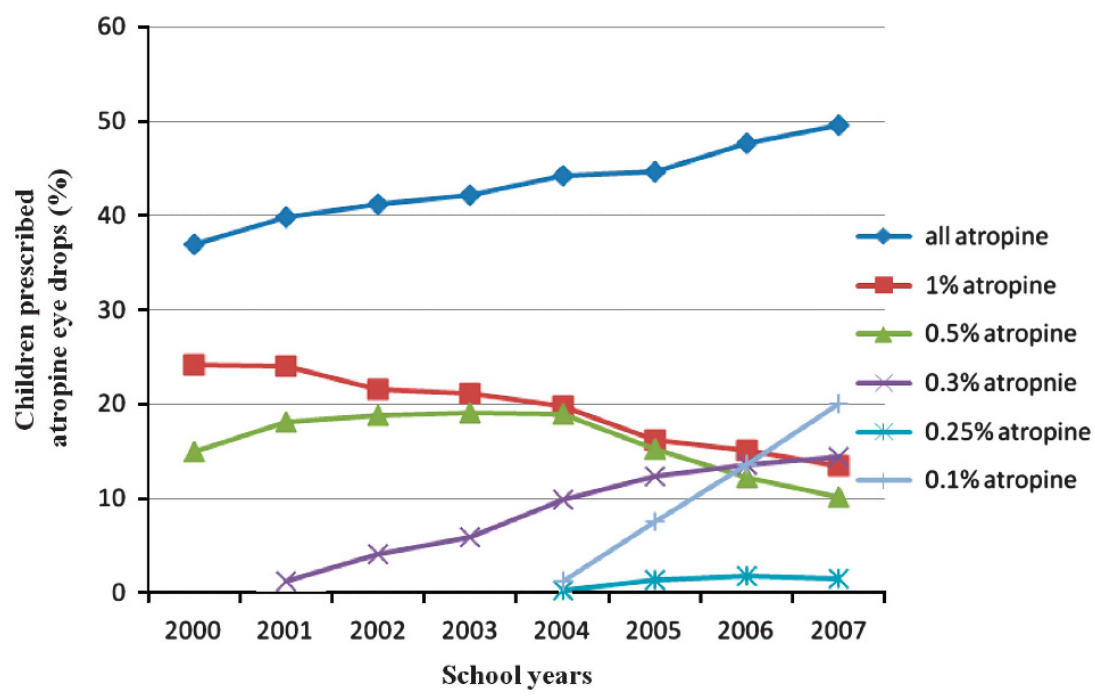

Figure 3 The trends of various concentrations of atropine eye drop prescription among 4-18-year-old children diagnosed with myopia (2000 to 2007).

increasing trend for atropine eye drop prescription (Figure 2). This figure shows an inverted U-shaped relationship between age and atropine prescription, with the highest rate for the 9-12 year olds. The trends associated with prescriptions of different concentrations of atropine eye drops are shown in Figure 3. The prescriptions of 0.5 and $1.0 \%$ atropine decreased after 2004, whereas the $0.3 \% / 0.25 \%$ atropine prescriptions increased steadily from 2001 and 2004 onwards, respectively. After 2004, there was a sharp increase in the prescription of $0.1 \%$ atropine.

The pattern of atropine prescription for different study variables is shown in Table 1 . The factors associated with atropine prescription for children diagnosed with myopia are listed in Table 2. Children aged 13-15 years $(\mathrm{OR}=0.73,95 \% \mathrm{CI}=0.71-0.75, P<0.0001)$ and $16-18$ years $(\mathrm{OR}=0.40,95 \% \mathrm{CI}=0.39-0.42, P<0.0001)$ had lower odds of being prescribed atropine eye drops compared with the younger primary school students (aged 7-8 years). Boys and girls were being prescribed atropine on an equal basis $(\mathrm{OR}=1.01,95 \% \mathrm{CI}=0.99-1.02$, $P=0.484$ ). Children of parents with a high SES (monthly wage NTD $\geq 40000$ ) had a higher odds ratio of being prescribed atropine $(\mathrm{OR}=1.22,95 \% \mathrm{CI}=1.19-1.25$, $P<0.0001$ ), whereas children whose parents or guardians were farmers or fishermen had a lower odds $(\mathrm{OR}=0.89$, 95\% CI 0.86-0.92, $P<0.0001)$ compared with children whose guardians had a monthly wage of NTD $<20000$. Children living in areas with mid to low levels of urbanization were less likely to be prescribed atropine compared with those living in high urbanization areas $(\mathrm{OR}=0.83$ and 0.67 respectively, both $P<0.0001)$.

\section{Discussion}

The NHI claims data allow us to investigate the trend of atropine prescription using a national representative sample of all individuals over a long period of time (2000-2007). Population-based information of drug utilization in such a large scale is rarely available in other countries. The high prevalence rate of myopia among Taiwanese children renders Taiwan a good candidate suitable for such investigation. This research identified the increasing trend of atropine eye drop prescription for school children aged 4-18 years who were diagnosed with myopia between 2000 and 2007, with a concurrent shift from prescribing high to low concentrations of atropine eye drops.

If our results are linked to previous epidemiological studies, the prevalence of myopia (cycloplegic refraction of less than $-0.25 \mathrm{D}),{ }^{8,21}$ remained relatively unchanged from 2000 to 2006 among 7-year-old students (20\% in 2000 and 2006), among 12-year-old students (61\% in 2000 and $62 \%$ in 2006) and among junior and senior high school students ( $\sim 76$ and $85 \%$, respectively). However, although the prevalence of myopia did not change a great deal, the rate for 4- to 18-year-old children visiting ophthalmologists increased significantly from $12.7 \%$ in 2000 to $20.1 \%$ in 2006. (Figure 1) This trend might be related to the annual vision screening of primary and secondary school students, as required by the Ministry of Education as part of the 'Enforcement of the Children Eye Health Act'. According to a technical report from the Ministry of Education in 2004, the trend of children who failed the vision screening to visit an ophthalmologist for further evaluation had increased from $84.7 \%$ in school 
Table 1 Atropine eye drop ${ }^{\text {a }}$ prescription (\%) among children diagnosed with myopia ${ }^{b}$ during school years 2000 to 2007 in Taiwan

\begin{tabular}{lcccc}
\hline Variables & & & & \\
School year & 2000 & 2004 & 2007 & $2000-2007$ \\
Number of children & 22417 & 32709 & 36058 & 240739 \\
Atropine eye drops (\%) & 36.9 & 44.2 & 49.5 & 43.9 \\
& & & & \\
Age group (\%) & & & & \\
4-6 & 28.1 & 31.2 & 35.1 & 31.6 \\
7-8 & 37.7 & 45.6 & 51.2 & 44.8 \\
9-10 & 44.8 & 52.2 & 59.7 & 52.9 \\
11-12 & 42.4 & 52.1 & 57.6 & 51.0 \\
13-15 & 31.1 & 38.1 & 41.6 & 37.2 \\
16-18 & 20.5 & 25.1 & 28.5 & 25.1 \\
& & & & \\
Gender (\%) & & & & \\
Boys & 37.1 & 44.4 & 49.4 & 44.0 \\
Girls & 36.8 & 44.1 & 49.7 & 43.7 \\
& & & & \\
Socioeconomic status (\%) & & & & \\
NTD $<20$ 000 & 36.5 & 43.8 & 49.1 & 43.4 \\
NTD 20 000-39999 & 36.4 & 43.8 & 49.2 & 43.5 \\
NTD $\geq 40$ 000 & 41.8 & 49.6 & 55.7 & 49.2 \\
Farmers and fishermen & 30.4 & 37.5 & 43.5 & 37.2 \\
Others & \\
& 34.1 & 40.4 & 44.4 & 40.3 \\
Urbanization level (\%) & & & & \\
Level 1 & & & & \\
Level 2 & 43.0 & 49.7 & 55.3 & 49.5 \\
Level 3 & 37.8 & 44.7 & 49.2 & 44.1 \\
\hline
\end{tabular}

To conserve space, only data of total year (2000 to 2007) and school years 2000, 2004, and 2007 are shown; data of other years are available on request.

${ }^{a}$ Atropine eye drops include: $1 \%$ atropine, $0.5 \%$ atropine, $0.3 \%$ atropine $0.25 \%$ atropine, and $0.1 \%$ atropine eye drop.

${ }^{\mathrm{b}}$ We used a broader definition of myopia (ICD-9-CM as 367) in this study. ${ }^{\mathrm{c}}$ NTD is New Taiwan Dollar (1 USD $\fallingdotseq 30$ NTD).

${ }^{\mathrm{d}}$ Others: low-income family or individuals who have no regular job.

year 2000 to $95.0 \%$ in school year 2003. While around $85 \%$ of the children who visited an ophthalmologist were diagnosed with ICD-9 CM 367 (the broader definition of myopia), the reason for consulting an eye doctor remained relatively unchanged during the study period. The odds for myopic children visiting an ophthalmologist in Taiwan to being prescribed atropine eye drops increased by $34 \%$ between $2000(36.9 \%)$ and 2007 (49.5\%). This substantial increase in atropine prescription might be because of the increased use of lower concentration of atropine, which produces a lower level of photophobia and near blurring. ${ }^{12}$ The rate of atropine prescriptions for all 4-18-year-old children in Taiwan increased 2.29-fold from $4.5 \%$ in 2000 to $10.3 \%$ in 2007 (table not shown). Therefore, the above evidences allow us to deduce that both the increasing rate of ophthalmologist visiting and the increasing rate of atropine prescription from ophthalmologist consist of the two key components of the increasing trend of atropine prescription for all children in Taiwan.
Table 2 Multiple logistic regression for factors associated with atropine eye drop ${ }^{\mathrm{a}}$ prescription $(n=240739)$

\begin{tabular}{|c|c|c|c|}
\hline & \multicolumn{3}{|c|}{ Atropine eye drop prescription } \\
\hline & $O R^{\mathrm{b}}$ & $95 \% C I$ & P-value \\
\hline \multicolumn{4}{|l|}{ Variables } \\
\hline \multicolumn{4}{|l|}{ Age } \\
\hline $4-6$ & 0.56 & $0.54-0.58$ & $<0.0001$ \\
\hline $7-8$ & 1.00 & & \\
\hline 9-10 & 1.38 & $1.35-1.42$ & $<0.0001$ \\
\hline $11-12$ & 1.29 & $1.26-1.32$ & $<0.0001$ \\
\hline $13-15$ & 0.73 & $0.71-0.75$ & $<0.0001$ \\
\hline $16-18$ & 0.40 & $0.39-0.42$ & $<0.0001$ \\
\hline \multicolumn{4}{|l|}{ Gender } \\
\hline Boy & 1.00 & & \\
\hline Girl & 1.01 & $0.99-1.02$ & 0.4843 \\
\hline \multicolumn{4}{|l|}{ Socioeconomic status } \\
\hline NTD $^{c}<20000$ & 1.00 & & \\
\hline NTD $20000-39999$ & 0.99 & $0.96-1.01$ & 0.2661 \\
\hline NTD $\geq 40000$ & 1.22 & $1.19-1.25$ & $<0.0001$ \\
\hline Farmers and fishermen & 0.89 & $0.86-0.92$ & $<0.0001$ \\
\hline Others $^{\mathrm{d}}$ & 0.86 & $0.84-0.89$ & $<0.0001$ \\
\hline \multicolumn{4}{|l|}{ Urbanization level } \\
\hline Level 1 & 1.00 & & \\
\hline Level 2 & 0.83 & $0.81-0.84$ & $<0.0001$ \\
\hline Level 3 & 0.67 & $0.65-0.68$ & $<0.0001$ \\
\hline
\end{tabular}

${ }^{a}$ Atropine eye drops include: $1 \%$ atropine, $0.5 \%$ atropine, $0.3 \%$ atropine, $0.25 \%$ atropine, and $0.1 \%$ atropine eye drop.

${ }^{\mathrm{b}}$ The OR represented the adjusted OR. The full model included the following variables: age groups, gender, socioeconomic status, urbanization level, and school years.

${ }^{\mathrm{c}}$ NTD is New Taiwan Dollar (1 USD $\fallingdotseq 30$ NTD).

${ }^{\mathrm{d}}$ Others: low-income family or individuals who have no regular job.

Most ophthalmologists would agree that age is an important consideration when treating myopia.

Although in most clinical studies atropine is used to treat 6-13-year-old children, ${ }^{11-15}$ in Taiwan, $41.6 \%$ of $13-15-$ year-old children (junior high school students) and 28.5\% of 16-18-year-old children (senior high school students) were still being prescribed atropine during 2007. The present study shows that atropine remains being prescribed to a certain portion of 13-18-year-old children, indicating that doctors are still concerned about myopia progression in these age groups. Different from studies in Hong Kong and Singapore school children that showed highest myopic progression in primary school students, ${ }^{22,23}$ Shih et $^{118}$ reported that junior high school students had a higher progression rate than primary school students and senior high school students in Taiwan. Further studies of this 13-18-year-old group are needed to reveal the effectiveness of atropine as a means of decreasing myopia progression.

Prior studies in Taiwan indicate that higher degree of urbanization is associated with the higher prevalence,, 24 
and higher progression rate of myopia. ${ }^{18}$ In the same token, myopia is highly associated with atropine prescription. In our study, we discovered that higher levels of urbanization were found to be significantly associated with a higher rate of atropine prescription when compared with a lower level of urbanization. It is possible that this is not only associated with the progression rate of myopia, ${ }^{18}$ but also with the severity of myopia, and/or the medical resources available in the various areas with different urbanization levels.

In our study, children of high SES families tended to be prescribed atropine more often. Studies have shown that adults with a high SES tend to be associated with a higher myopic refraction. ${ }^{25}$ It is well known that the refractive status of parents influences their children's myopia ${ }^{26}$ through either hereditary or environmental factors. ${ }^{27-29}$ A strong parental history of myopia is associated with faster eye growth and a myopic shift. ${ }^{30}$ Therefore, children from a high socioeconomic background may have a higher prevalence of myopia and faster myopic progression, which justifies a higher level of prescription of atropine. Alternatively, these children may also have a weaker economic barrier to ophthalmology outpatient visits and thus have a greater chance of being treated.

This study also has some limitations, which must be borne in mind while interpreting the study result. First, although dilution of the atropine eye drops by medical institutions is not allowed under NHI regulations, we still cannot neglect the possible cases of drug dilutions. Lee $e a^{31}$ pointed out that using $0.05 \%$ atropine eye drops is still effective in retarding the rate of myopia progression. It is possible that some institutions prescribe high concentration of eye drops for patients, but which are then diluted to a lower concentration in practice. Nevertheless, this should not result in significant bias if we assume that this phenomenon is constant across the years. In addition, if dilution was considered, only high concentrations of eye drops can be diluted to low concentration ones, and not the reverse. Thus, the shift from high to low concentration of atropine for actual use would be more significant if the actual concentration would be measured. Second, we are unable to measure drug compliance, which is beyond the scope of this study. Poor compliance is an important issue for all chronic diseases. Our data are reimbursed records; thus, it only shows the trend for prescribing atropine eye drops by ophthalmologists. Finally, other treatment modalities such as multifocal lenses, contact lenses, orthokeratology, acupuncture, or traditional medicine used for halting myopia progression are not covered under the NHI program, and thus cannot be evaluated in this study. Consequently, we could not determine whether there were combined uses or other treatments that involved these treatment modalities.

This study concludes that a number of ophthalmologists in Taiwan considered that this drug could be used to retard myopia progression in children. The increased prescription trend might also be related to a greater concern by parents about their children's eye health and the availability of lower concentration of atropine eye drops. Further studies on the long-term effects of atropine on the retina and lens are still needed. We will like to reinforce that the use of atropine among children 13-18 years of age should be based on clinically supported evidence rather than mere speculation.

Nonetheless, based on this study, other governments and policy makers worldwide are now in a better position when considering the integration of atropine as a treatment modality in their countries.

\section{Summary}

What is known about this topic

- Atropine is a modality for halting myopia progression in children.

- Although higher concentrations of atropine eye drops are associated with better efficacy, clinical studies have shown that those who are prescribed higher concentration experience higher withdrawal rate.

What this study adds

- The real prescription pattern of atropine eye drops increased from 2000 to 2007 in Taiwan; and the prescription of atropine eye drops shifted from high to low concentration during this period.

- Atropine eye drops were more frequently prescribed to 9-12-year-old children, children living in highly urbanized areas, and children from families with a high socioeconomic status.

\section{Conflict of interest}

The authors declare no conflict of interest.

\section{Acknowledgements}

This study was not supported by any government or non-government institutions. This submission has not been published anywhere previously and it is not simultaneously being considered for any other publication.

\section{References}

1 Lam CS, Goldschmidt E, Edwards MH. Prevalence of myopia in local and international schools in Hong Kong. Optom Vis Sci 2004; 81(5): 317-322. 
2 Saw SM, Wu HM, Seet B, Wong TY, Yap E, Chia KS et al. Academic achievement, close up work parameters, and myopia in Singapore military conscripts. $\mathrm{Br} J$ Ophthalmol 2001; 85(7): 855-860.

3 Matsumura $\mathrm{H}$, Hirai $\mathrm{H}$. Prevalence of myopia and refractive changes in students from 3 to 17 years of age. Surv Ophthalmol 1999; 44(Suppl 1): S109-S115.

4 Bar Dayan Y, Levin A, Morad Y, Grotto I, Ben-David R, Goldberg A et al. The changing prevalence of myopia in young adults: a 13-year series of population-based prevalence surveys. Invest Ophthalmol Vis Sci 2005; 46(8): 2760-2765.

5 Vitale S, Sperduto RD, Ferris 3rd, FL. Increased prevalence of myopia in the United States between 1971-1972 and 19992004. Arch Ophthalmol 2009; 127(12): 1632-1639.

6 Villarreal MG, Ohlsson J, Abrahamsson M, Sjostrom A, Sjostrand J. Myopisation: the refractive tendency in teenagers. Prevalence of myopia among young teenagers in Sweden. Acta Ophthalmol Scand 2000; 78(2): 177-181.

7 He M, Zeng J, Liu Y, Xu J, Pokharel GP, Ellwein LB. Refractive error and visual impairment in urban children in Southern China. Invest Ophthalmol Vis Sci 2004; 45(3): 793-799.

8 Lin LL, Shih YF, Hsiao CK, Chen CJ. Prevalence of myopia in Taiwanese schoolchildren: 1983 to 2000. Ann Acad Med Singapore 2004; 33(1): 27-33.

9 Saw SM, Gazzard G, Au Eong KG, Tan DT. Myopia: attempts to arrest progression. Br J Ophthalmol 2002; 86(11): 1306-1311.

10 Saw SM, Shih-Yen EC, Koh A, Tan D. Interventions to retard myopia progression in children: an evidence-based update. Ophthalmology 2002; 109(3): 415-421; discussion 422-4; quiz 425-6, 443.

11 Yen MY, Liu JH, Kao SC, Shiao CH. Comparison of the effect of atropine and cyclopentolate on myopia. Ann Ophthalmol 1989; 21(5): 180-182; 187

12 Shih YF, Chen CH, Chou AC, Ho TC, Lin LL, Hung PT. Effects of different concentrations of atropine on controlling myopia in myopic children. J Ocul Pharmacol Ther 1999; 15(1): 85-90.

13 Shih YF, Hsiao CK, Chen CJ, Chang CW, Hung PT, Lin LL. An intervention trial on efficacy of atropine and multi-focal glasses in controlling myopic progression. Acta Ophthalmol Scand 2001; 79(3): 233-236.

14 Chua WH, Balakrishnan V, Chan YH, Tong L, Ling Y, Quah BL et al. Atropine for the treatment of childhood myopia. Ophthalmology 2006; 113(12): 2285-2291.

15 Fan DS, Lam DS, Chan CK, Fan AH, Cheung EY, Rao SK. Topical atropine in retarding myopic progression and axial length growth in children with moderate to severe myopia: a pilot study. Jpn J Ophthalmol 2007; 51(1): 27-33.

16 Tong L, Huang XL, Koh AL, Zhang X, Tan DT, Chua WH. Atropine for the treatment of childhood myopia: effect on myopia progression after cessation of atropine. Ophthalmology 2009; 116(3): 572-579.
17 Chia A, Chua WH, Cheung YB, Wong WL, Lingham A, Fong A et al. Atropine for the treatment of childhood myopia: safety and efficacy of $0.5 \%, 0.1 \%$, and $0.01 \%$ doses (Atropine for the Treatment of Myopia 2). Ophthalmology 2012; 119(2): 347-354.

18 Shih YF, Chiang TH, Hsiao CK, Chen CJ, Hung PT, Lin LL. Comparing myopic progression of urban and rural Taiwanese schoolchildren. Jpn J Ophthalmol 2010; 54(5): 446-451.

19 Shen HN, Lu CL, Yang HH. Epidemiological trend of severe sepsis in Taiwan from 1997 through 2006. Chest 2010; 138(2): 298-304.

20 Wu CY, Hu HY, Huang N, Pu CY, Shen HC, Chou YJ. Do the health-care workers gain protection against herpes zoster infection? A 6-year population-based study in Taiwan. J Dermatol 2010; 37(5): 463-470.

21 Bureau of Health Promotion, Department of Health, R.O.C. Epidemiology of myopia among 6 to 18 year old children in Taiwan [in Chinese]. Technical Report 2008.

22 Lam CS, Edwards M, Millodot M, Goh WS. A 2-year longitudinal study of myopia progression and optical component changes among Hong Kong schoolchildren. Optom Vis Sci 1999; 76(6): 370-380.

23 Saw SM, Tong L, Chua WH, Chia KS, Koh D, Tan DT et al. Incidence and progression of myopia in Singaporean school children. Invest Ophthalmol Vis Sci 2005; 46(1): 51-57.

24 Guo YH, Lin HY, Lin LL, Cheng CY. Self-reported myopia in Taiwan: 2005 Taiwan National Health Interview Survey. Eye (Lond) 2012; 26(5): 684-689.

25 Wong TY, Foster PJ, Johnson GJ, Seah SK. Education, socioeconomic status, and ocular dimensions in Chinese adults: the Tanjong Pagar Survey. Br J Ophthalmol 2002; 86(9): 963-968.

26 Goss DA, Jackson TW. Clinical findings before the onset of myopia in youth: 4. Parental history of myopia. Optom Vis Sci 1996; 73(4): 279-282.

27 Wu MM, Edwards MH. The effect of having myopic parents: an analysis of myopia in three generations. Optom Vis Sci 1999; 76(6): 387-392.

28 Mutti DO, Mitchell GL, Moeschberger ML, Jones LA, Zadnik K. Parental myopia, near work, school achievement, and children's refractive error. Invest Ophthalmol Vis Sci 2002; 43(12): 3633-3640.

29 Zadnik K, Satariano WA, Mutti DO, Sholtz RI, Adams AJ. The effect of parental history of myopia on children's eye size. JAMA 1994; 271(17): 1323-1327.

30 Lam DS, Fan DS, Lam RF, Rao SK, Chong KS, Lau JT et al. The effect of parental history of myopia on children's eye size and growth: results of a longitudinal study. Invest Ophthalmol Vis Sci 2008; 49(3): 873-876.

31 Lee JJ, Fang PC, Yang IH, Chen CH, Lin PW, Lin SA et al. Prevention of myopia progression with $0.05 \%$ atropine solution. J Ocul Pharmacol Ther 2006; 22(1): 41-46. 